\title{
BMJ Open Effects of high-load and low-load resistance training in patients with coronary artery disease: rationale and design of a randomised controlled clinical trial
}

Tim Kambic (10 , ${ }^{1}$ Nejc Šarabon,,${ }^{2,3,4}$ Vedran Hadžić, ${ }^{5}$ Mitja Lainscak ${ }^{6,7,8}$

To cite: Kambic T, Šarabon N, Hadžić $\mathrm{V}$, et al. Effects of high-load and low-load resistance training in patients with coronary artery disease: rationale and design of a randomised controlled clinical trial. BMJ Open 2021;11:e051325. doi:10.1136/ bmjopen-2021-051325

- Prepublication history and additional supplemental material for this paper are available online. To view these files, please visit the journal online. To view these files, please visit the journal online (http://dx.doi org/10.1136/bmjopen-2021 051325).

Received 16 March 2021 Accepted 01 July 2021

Check for updates

(C) Author(s) (or their employer(s)) 2021. Re-use permitted under CC BY-NC. No commercial re-use. See rights and permissions. Published by BMJ.

For numbered affiliations see end of article.

Correspondence to

Prof. Mitja Lainscak;

mitja.lainscak@guest.arnes.si

\section{ABSTRACT}

Introduction Resistance training (RT) combined with aerobic training (AT) enhances the effects of cardiac rehabilitation (CR) in patients with coronary artery disease (CAD). However, it remains to be investigated which type of RT (high loads (HLs) vs low loads (LLs)) is more efficacious in improving exercise performance, cardio-metabolic health and quality of life.

Methods and analysis A randomised, controlled, clinical trial will enrol 20 patients with CAD into each of three study arms (total 60 patients): HL-RT ( $70 \%-80 \%$ of one repetition maximum (1-RM)) combined with AT; LL-RT (30\% $-40 \%$ of $1-\mathrm{RM})$ combined with AT and AT alone as standard care. Primary outcomes (maximal aerobic capacity, maximal leg isometric strength) will be assessed at baseline and after 36 training sessions. Other outcomes will include acute haemodynamic responses to LL-RT and HL-RT, body composition, physical performance, blood biomarkers (lipids, glucose metabolism, inflammation, growth factors), physical activity and quality of life. The intention-to-treat principle will be used to analyse the data. Ethics and dissemination The study design and protocol have been approved by the National Medical Ethics Committee of Slovenia (registration number: 0120$573 / 2019 / 15)$. The study will be conducted in accordance with the Declaration of Helsinki. The results of the study will be published as peer-reviewed manuscripts and congress presentations, communicated with patients and the clinical community, and shared through posts on social media. The findings of the study will be disseminated among the national CR clinical community (CR centres, Slovenian association of coronary clubs) with active participation of the patients enrolled in the study. This study will expand our knowledge of RT in combination with AT in CR. We expect to find different effects of HL$R T$ versus $L L-R T$, with implications for RT strategies in rehabilitation of patients with CAD.

Trial registration number NCT04638764.

\section{INTRODUCTION}

Cardiac rehabilitation (CR) is a standard multidisciplinary intervention for treatment and secondary prevention of cardiovascular diseases. The usual components of
Strengths and limitations of this study

- This study will be the first to evaluate the potential dose-dependent relationship of resistance training (RT) (high load (HL)-RT vs low load (LL)-RT) combined with aerobic training in patients with coronary artery disease.

- The study will evaluate safety and haemodynamic responses to $\mathrm{HL}$-resistance exercise (RE) $(80 \%$ of repetition maximum (1-RM)) and to LL-RE (40\% of $1-\mathrm{RM})$ in patients in a crossover, randomised and load balanced manner at inclusion to the cardiac rehabilitation (CR) programme.

- The study will implement progressive RT programmes, with balanced training volume, by the number of repetitions in LL-RT and HL-RT.

- The structure and progression of HL-RT and LL-RT follows a standard CR scheme, which allows the findings to be immediately translated to clinical practice.

- A potential limitation of the study is that the training load in both RT groups cannot be blinded within the randomly allocated cluster of patients.

CR are aerobic exercise training and physical activity advice, risk-factor screening and education, stress management, psychological support and optimal pharmacological treatment according to centre availability and resources. ${ }^{1} \mathrm{CR}$ is associated with improvement in aerobic capacity, muscle strength and quality of life, ${ }^{12}$ all of which lead to reduced rates of hospitalisation and cardiovascular mortality. ${ }^{3}$ The core component of CR is exercise training, wherein aerobic training (AT) is predominately recommended. Resistance training (RT) is underused in clinical practice, ${ }^{5}$ despite being recommended for over 20 years for patients with coronary artery disease (CAD). ${ }^{16-8}$ Implementation of RT is still limited by the lack of consensus among leading CR organisations worldwide ${ }^{5}$ and by 
the heterogeneous training protocols reported in individual randomised studies, ${ }^{29}$ as well as by safety concerns associated with cardiovascular response during exertion. ${ }^{7}$

Until very recently, ${ }^{1}$ only progressive low-to-moderate load RT $(30 \%-60 \%$ of one repetition maximum (1-RM)) was recommended for patients enrolled in $\mathrm{CR} .{ }^{7}$ Training stimuli in low-load (LL) RT may often be suboptimal for increase in muscle strength when compared with highload (HL) RT ( $>70 \%$ of $1-\mathrm{RM})$, as is recommended for both healthy young and older adults. ${ }^{10}$ Thus, in young athletes and the elderly, HL-RT has induced greater increase in muscle strength than LL-RT. ${ }^{11} 12$ In patients with CAD already experienced in CR training, HL resistance exercise (RE) (70\%-90\% 1-RM) is proven to be safe, with lower haemodynamic responses (eg, heart rate, blood pressure, cardiac output) and lower perceived exertion (eg, Borg scale) than observed in LL-RE (30\%$40 \%$ of $1-\mathrm{RM}) .{ }^{1314}$

To date, previous exercise interventions have demonstrated a greater effect of combined RT with AT on aerobic capacity, ${ }^{9}$ peak work capacity, lower-body strength and body composition when compared with AT alone in patients with CAD. ${ }^{29}$ However, no study has investigated the safety and efficacy of HL-RT ( $>70 \%$ of 1-RM) in comparison with LL-RT ( $<40 \% 1 \mathrm{RM})$ in such patients. Therefore, our study has two aims. The primary aim is to examine the effects of HL-RT and LL-RT combined with AT in comparison with standard care (AT) on aerobic capacity and maximal muscle strength in patients with CAD. The secondary aim is to examine the effects of HL-RT and LL-RT combined with AT in comparison with standard care (AT) on blood biomarkers, physical activity and quality of life. In addition, this study will compare the safety and acute haemodynamic responses to LL-RE and HL-RE at baseline and after 36 training sessions.

\section{METHODS AND ANALYSIS \\ Study design}

The study is designed as a randomised, controlled, clinical trial with three parallel intervention arms (figure 1): HL-RT combined with aerobic interval training; LL-RT combined with aerobic interval training and aerobic interval training as standard care. The design of the study accords with Consolidated Standards of Reporting Trials guidelines. ${ }^{15}$ After baseline clinical assessment, patients will be allocated into the three groups, using cluster randomisation (sealed envelope for each randomised cluster) prepared by an experienced epidemiologist who has no relation to the study procedures. Each cluster will comprise five patients $(5: 5: 5)$. The patients will not be blinded during the study; however, each cluster of randomised patients will train separately to avoid additional comparison with other interventional arms.

The primary outcomes of the study are change of maximal aerobic capacity $(\mathrm{mL} / \mathrm{kg} / \mathrm{min})$ and change of maximal isometric strength of knee extensors (newton metre, Nm). Secondary outcomes include assessment of physical performance (muscle strength and endurance, flexibility, postural balance), haemodynamics during exercise, anthropometry and body composition, blood markers (glucose metabolism, blood lipids, etc), subjectively measured physical activity and quality of life (table 1).

Measurements will be made at baseline and after 36 CR training sessions (figure 1). Baseline and post-CR data will each be collected on two separate occasions with at least 48 hours rest between measurement days. At baseline, all patients will have an extensive medical examination by a cardiologist, donate a blood sample at rest and then complete a cardiopulmonary exercise test (CPET). After at least 48 hours rest, patients' body composition will be measured together with physical performance tests (assessing whole-body strength, postural balance, flexibility), and patients will be familiarised with RT on a legpress machine. Patients will complete questionnaires on quality of life and physical activity, and will receive accelerometers for 8 days of monitoring. Afterwards, patients will be allocated to each of the three study groups.

CR follows a standard scheme of three sessions per week for 12 weeks. Before the first session each week, patients will complete a 1-RM test on a leg-press machine followed by AT. Before the second and third sessions, haemodynamic responses (measured as change in heart rate and blood pressure) to HL-RE and LL-RE will be evaluated. Patients will then perform exercise training according to their group allocation. They will be permitted to continue with low-to-moderate intensity physical activity at home during the rest days (eg, walking, cycling, callisthenics, etc), with exception of resistance exercise. At week 8, legpress strength will be re-evaluated and RT load adjusted accordingly. Within 2-5 days after session 36 , all baseline measurements will be repeated.

\section{Participants}

Patients with stable CAD (acute coronary syndrome and/ or percutaneous coronary intervention) will be recruited from the Division of Cardiology, General Hospital Murska Sobota, Slovenia. Inclusion criteria are: age $18-85$ years, left ventricular ejection fraction $\geq 40 \%$, documented CAD and completion of a baseline CPET. ${ }^{1}$ Exclusion criteria follow previous recommendations for participation in RT (box 1). ${ }^{8}$ Recruitment of patients began in July 2020 and is expected to be completed in July 2021.

\section{Training protocol}

Patients will complete a total of 36 training sessions (three training sessions per week for 12 weeks or until completion, with at least 48 hours rest between sessions). Each training session will consist of general warm-up (10 min dynamic flexibility exercises followed by callisthenics using elastic bands and/or LL dumbbells and balance exercises), aerobic interval cycling and RT (60 min), and cool down (5 min static stretching and breathing exercises). All patients will perform aerobic interval cycling (3-5 min workload cycling separated by 2 min unloaded 


\section{Baseline measurements}

Visit 1:

1. Diagnostic screening

2. Blood sample

3. CPET
Visit 2:

1. Anthropometry and body composition

2. MVC and evaluation of physical performance

3. Familiarization with leg press exercise

4. Questionnaires (IPAQ short form, PHQ-9, SF-12)

5. Physical activity monitoring (Actigraph monitor)

\section{Exercise training intervention}

(36 exercise sessions)

\section{1-RM test}

Haemodynamic response to $H L$ and $L L R E$

Exercise session: 1, 22 and 36

Exercise session: 2- 3 and 34- 35

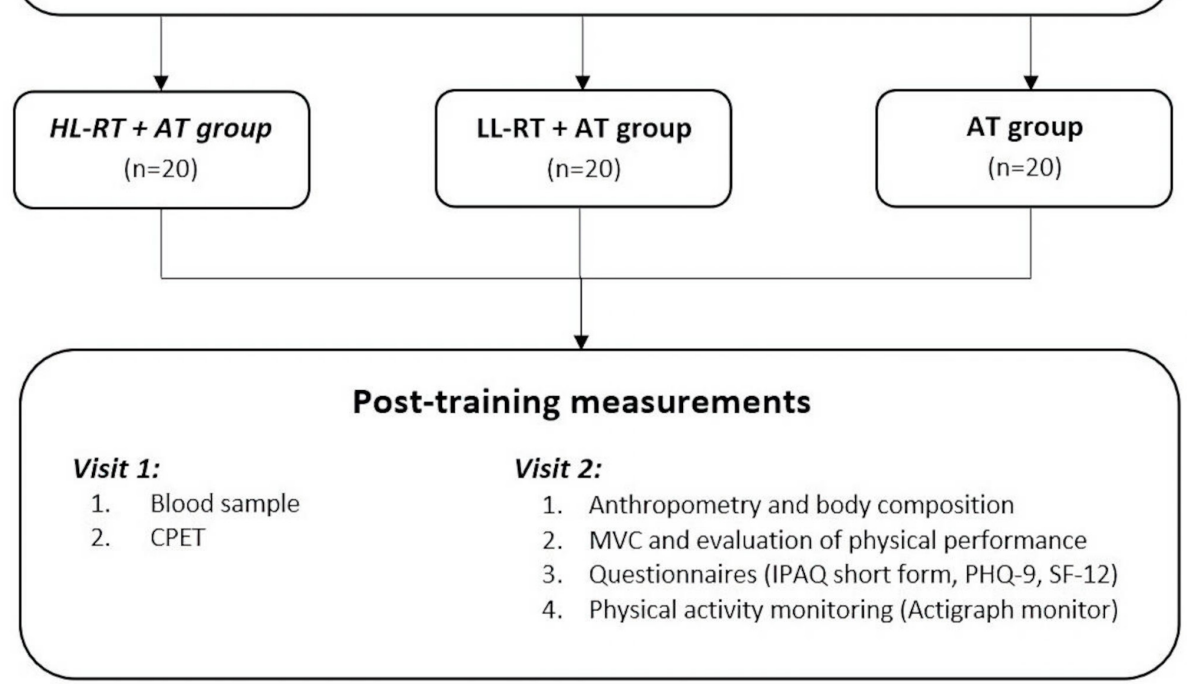

Figure 1 Overview of study design and measurements. AT, aerobic training; CPET, cardiopulmonary exercise test; HL, high load; LL, low load; IPAQ, International Physical Activity Questionnaire; MVC, maximal voluntary contraction; PHQ-9, Patient Health Questionnaire-9; RE, resistance exercise; 1-RM, one repetition maximum; RT, resistance training; SF-12, 12-Item Short Form Survey.

cycling) starting from the initial $50 \%$ of maximal workload (Pmax) achieved at baseline CPET and progressively increasing every 2 weeks to $80 \%$ Pmax (table 2). Cycling cadence will be set at $50-60$ revolutions $/ \mathrm{min}^{1}$

In both RT groups, each patient will complete a total of 36 sessions on a leg-press machine (3 1-RM tests and 33 RT sessions). The training load will differ between the two RT groups, whereas the training volume will be balanced by the number of repetitions (table 2). The range of number of repetitions accords with previous RT recommendations in CR. ${ }^{17}$ Patients will be familiarised with RT prior to baseline testing to ensure the use of correct lifting and breathing techniques and aiming to avoid the Valsalva manoeuvre. ${ }^{78}$ In the HL-RT group, workload will be increased from an initial three sets at intensity $70 \%$ of 1-RM (6-11 repetitions per set) to $80 \%$ of 1-RM (6-8 repetitions per set) in the first 7 weeks of the CR. In the
LL-RT group, workload will be increased from the initial $35 \%$ of 1-RM (12-22 repetitions per set) to $40 \%$ of 1-RM (12-16 repetitions per set). After 8 weeks of training, 1-RM will be re-evaluated in all three groups and the new maximal value will be used to prescribe RT for the final 4 weeks of CR. Thus, the load in the HL-RT group will progress from $70 \%$ 1-RM (11 repetitions per set) to $80 \%$ 1-RM (6-8 repetitions per set), and the load the in LL-RT group will progress from 35\% 1-RM (22 repetitions per set) to $40 \%$ 1-RM (12-16 repetitions per set). ${ }^{16-18} \mathrm{~A}$ lifting cadence of $1 \mathrm{~s}: 1 \mathrm{~s}$ (concentric and eccentric contraction) will be used, with $90 \mathrm{~s}$ rest between sets. ${ }^{19}$

Patients will be continuously monitored with beat-tobeat telemetry to monitor heart rate and blood pressure before, during and after each training modality. All training sessions will be supervised by a medical nurse and physiotherapist and guided by a kinesiologist, with 


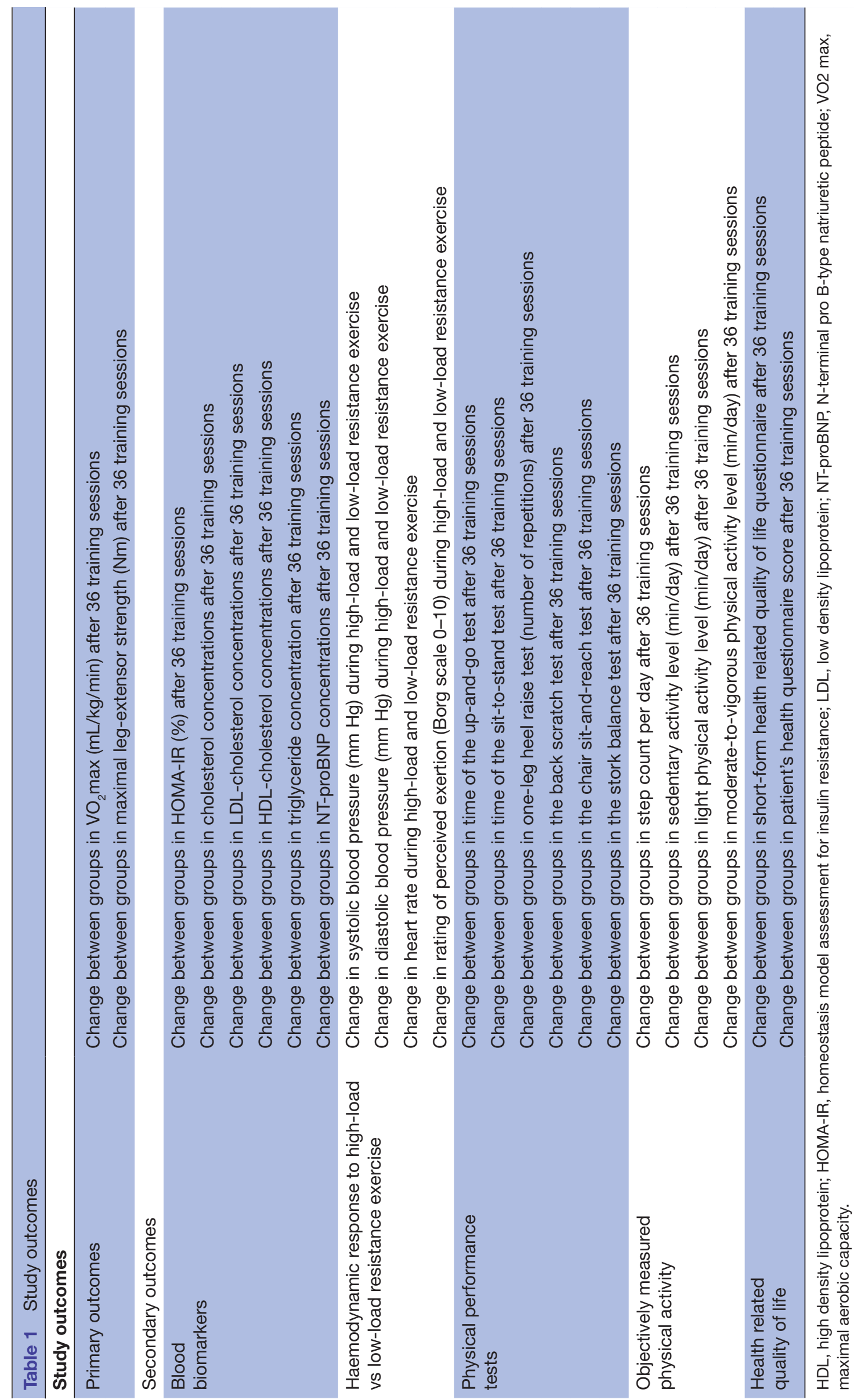


Box 1 Exclusion criteria for participation in resistance training

Absolute
Unstable angina pectoris.
Uncontrolled hypertension.
Systolic blood pressure $\geq 180 \mathrm{~mm} \mathrm{Hg}$.
Diastolic blood pressure $\geq 110 \mathrm{~mm} \mathrm{Hg}$.
Uncontrolled dysrhythmias including sinus tachycardia.
Recent history of congestive heart failure (not yet evaluated and
treated).
Severe stenotic or regurgitant valvular disease.
Hypertrophic cardiomyopathy.
Severe pulmonary hypertension (mean pulmonary arterial pressure
$>55$ mm Hg).
Acute myocarditis, endocarditis or pericarditis.
Aortic syndrome or venous thromboembolism.
Acute systemic illness.
Postural hypotension ( $\geq 20$ mm Hg drop in systolic blood pressure
with symptoms of dizziness or light-headedness).
Aortic dissection.
Recent embolism.
Thrombophlebitis.
Relative
Cognitive impairment.
Neural disease.
Musculoskeletal limitations and previous injuries (acute and chronic
injuries of lower back and lower limbs).

a cardiologist available for consultations on site. The medical staff will document any potential cardiovascular (blood pressure $>220 / 110 \mathrm{~mm} \mathrm{Hg}$, orthostatic intolerance, atrial fibrillation, arrhythmias, shortness of breath, dizziness, chest pain, etc) and musculoskeletal (muscle and joint pain, muscle fatigue) adverse symptoms and signs during and after each training session. All major adverse events will be evaluated for potential safety indications by the data management board within the Division of Cardiology at the General Hospital Murska Sobota. The data management board will consist of experienced consultant cardiologists and medical nurses. During screening of the potential major event, the patient will not participate in any activity within the CR programme and will resume training only on medical clearance.

\section{Measurements}

\section{Cardiopulmonary exercise testing}

Maximal aerobic capacity will be measured using an adjusted ramp protocol ${ }^{20}$ on a Schiller ERR 911 ergometer and a Cardiovit CS-200 excellence ergo-spirometer (Schiller, Baar, Switzerland). In brief, after receiving short instructions and completing a spirometry test, patients will be seated for determination of their baseline heart rate, blood pressure and gas exchange. Patients will then start cycling without workload for $3 \mathrm{~min}$ at 50-60 rpm, followed by increasing workload every minute for an additional $10-25 \mathrm{~W}$ until exhaustion or other contraindications.
Maximum voluntary isometric strength of knee extensors

Maximum isometric leg strength will be measured on a knee dynamometer (S2P, Ljubljana, Slovenia). Patients will be seated with their hips at $90^{\circ}$ flexion, knee at $60^{\circ}$ flexion (fully extended knee $0^{\circ}$ ), arms holding the handles and the trunk fixed with a seat belt system. The shank brace will be positioned on the distal one-third of the lower leg, two fingers above the ankle. The patient's legs will be tested simultaneously, with patients instructed to push their legs as fast and hard as possible and to hold the contraction for at least $3 \mathrm{~s}$. There will be breaks of $60 \mathrm{~s}$ between three maximum voluntary contractions (MVCs) ${ }^{21}$ Torque signals from the sensor will be collected at $1000 \mathrm{~Hz}$ and analysed using ARS analysis and reporting software (S2P). The highest torque value from three MVCs measured on a $1 \mathrm{~s}$ time interval will be included in further statistical analysis.

\section{Prediction and measurement of maximal leg-press strength}

Leg-press familiarisation and submaximal strength tests will be completed using a Life Fitness Leg Press Pro 2 (Life Fitness, Rosemont, Illinois, USA). After a general warm-up (5 min cycling at 50\% maximum heart rate with cadence 50-60 revolutions/min and dynamic stretching of lower limbs), patients will be shown the correct lifting technique and will be familiarised with the protocol for leg-press testing. Patients will be in a seated position with their back in permanent contact with the machine during the test, with hands holding the handles of the machine, and hips and knee at $90^{\circ}$ of flexion in the starting position. During the test, patients will complete a warm-up set comprising eight and six repetitions at $50 \%$ and $70 \%$ of their perceived 1-RM, respectively. The weight will be progressively increased until reaching the workload that can be lifted three to five times (3-5 RM), with 2-3 min rest between the trials. ${ }^{17}$ The $1-\mathrm{RM}$ will be calculated using the established 1-RM prediction equation, which is based on the maximal weight lifted and number of repetitions during the submaximal strength test (predicted 1-RM=maximal load lifted/1.0278-0.0278 $\times$ number of repetitions). ${ }^{22}$

\section{Haemodynamic responses during resistance exercise}

Response of heart rate and blood pressure to RE will be measured using an OMRON HBP 1320 professional BP monitor (Omron Healthcare, Vernon Hills, Illinois, USA) and a Nellcor Oximax N-65 pulse oximeter (Covidien, Manfield, Massachusetts, USA). Perceived exertion will be assessed using the short version of the Borg scale. ${ }^{23}$ Safety of patients will be ensured with continuous monitoring for any adverse cardiovascular signs (blood pressure $>220 / 110 \mathrm{~mm} \mathrm{Hg}$, orthostatic intolerance, atrial fibrillation, arrhythmias, etc), and they will be asked to report any cardiopulmonary (shortness of breath, dizziness, chest pain) and musculoskeletal (muscle and joint pain, muscle fatigue) adverse symptoms and signs after each set of RE. 
Table 2 Progression of aerobic and resistance training

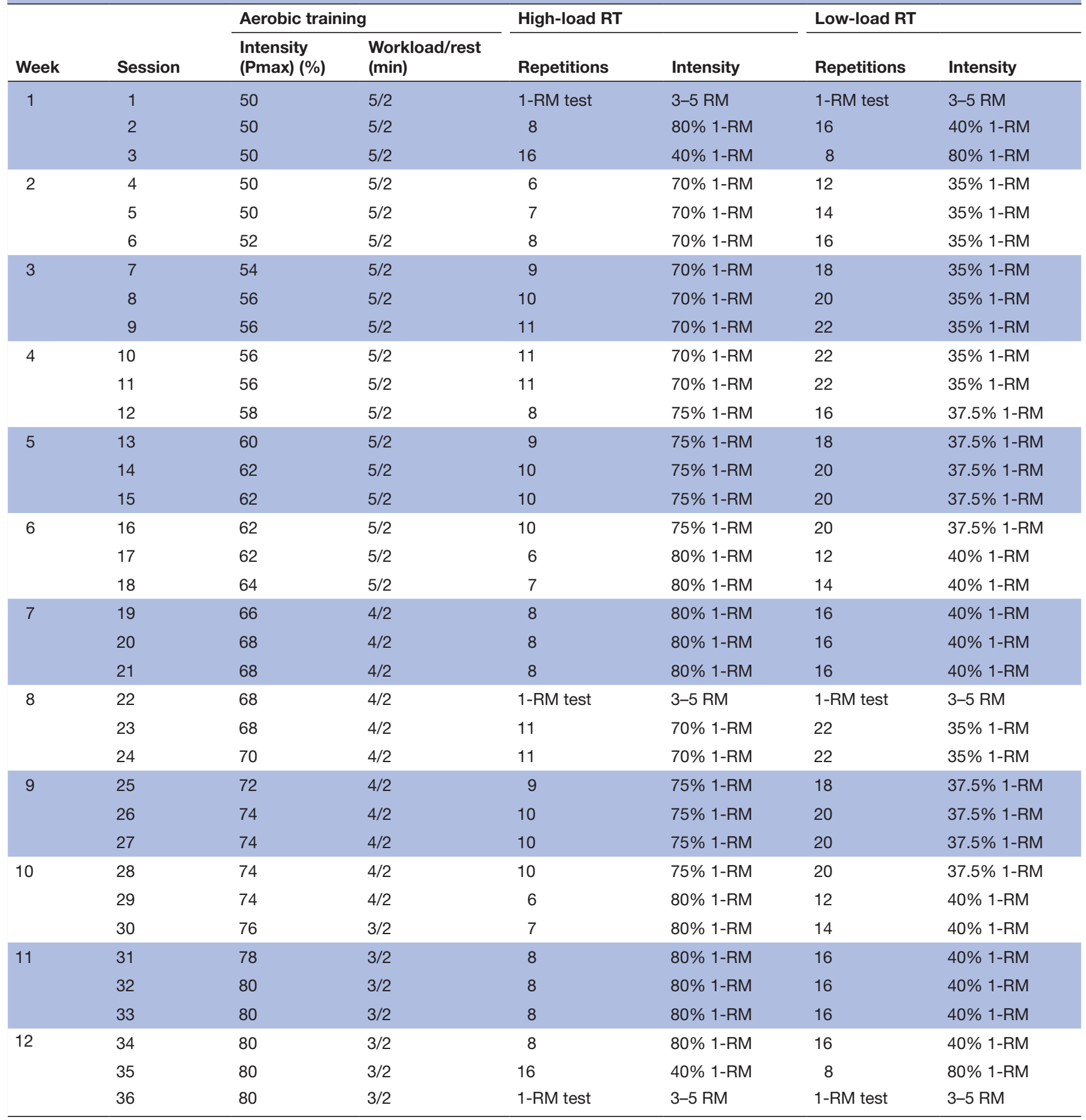

Pmax, maximal power output; 1-RM, one repetition maximum; RT, resistance training.

Patients will perform HL-RE and LL-RE at sessions 2 and 3 in a crossover, randomised manner. The same randomised order will be repeated at sessions 34 and 35 (figure 1). Haemodynamic parameters will be measured with patients in a seated position at baseline (after 3 min rest), again after the last repetition of each set, and lastly post-exercise (3 $\mathrm{min}$ post-exercise). After a general warm-up and baseline haemodynamic measurements, patients will perform RE according to their group allocation. RE will consist of three sets of either 16 repetitions at $40 \%$ of 1-RM (LL-RE) or 8 repetitions at $80 \%$ of 1-RM (HL-RE), with a lifting cadence ratio $1 \mathrm{~s}$ of concentric contraction and $1 \mathrm{~s}$ of eccentric contraction, and with 90 s rest between sets. ${ }^{131419}$ The cumulative load $(\mathrm{kg})$ will be balanced in both types of RE to eliminate the potential effects of training load. The same RE protocol using other resistance loads will be completed after a rest of 48-72 hours. 
Table 3 Physical performance battery

\begin{tabular}{|c|c|c|}
\hline Motoric ability & Outcome & Purpose \\
\hline \multicolumn{3}{|l|}{ Flexibility } \\
\hline Back-scratch test & Distance between middle fingers of hand $(\mathrm{cm})$ & Upper-body/shoulder flexibility \\
\hline Chair sit-and-reach test & $\begin{array}{l}\text { Distance between extended } \\
\text { middle fingers and tip of toes (cm) }\end{array}$ & Lower-body flexibility \\
\hline Arm curl test & Number of biceps curls using dumbbell & Upper-limb strength \\
\hline Hand grip test & Force of grip (kg) & Upper-limb strength \\
\hline Sit-to-stand test & Time of 5 and 10 sit-to-stands (s) & Lower-body strength \\
\hline One-leg heel raise test & Number of one-leg heel raises & Lower-body strength \\
\hline Timed up-and-go test & Time of the test (s) & Agility and dynamic balance \\
\hline \multicolumn{3}{|l|}{ Balance } \\
\hline SPPB feet together test & Time (s), up to $10 \mathrm{~s}$ & Balance \\
\hline SPPB semi-tandem test & Time (s), up to $10 \mathrm{~s}$ & Balance \\
\hline SPPB full tandem test & Time (s), up to $10 \mathrm{~s}$ & Balance \\
\hline Stork test & Time of the test (s) & Balance of lower limbs \\
\hline Stork test on balance pad & Time of the test (s) & Balance of lower limbs \\
\hline \multicolumn{3}{|l|}{ Endurance } \\
\hline 6 min walk test & 6 min walk distance $(\mathrm{m})$ & Whole-body endurance \\
\hline
\end{tabular}

SPPB, short physical performance battery.

\section{Physical performance}

At baseline and post-CR, patients will undergo extensive assessments of physical performance to evaluate muscle strength, endurance, flexibility and postural balance, using a hand-grip strength test, five repetitions of sitto-stand test, arm curl test, timed up-and-go test, chair sit-and-reach test, back scratch test, $4 \mathrm{~m}$ gait-speed test, single-leg standing test (stork test), one-heel rise test and 6 min walk test (table 3). Extensive description of the selected tests can be found elsewhere. ${ }^{24} 25$

\section{Anthropometry and body composition}

Body height and weight will be measured on a Marsden DP3810 weighing scale and stadiometer (Marsden Weighing Group, Rotherham, UK); waist and hip circumference will be measured with a standard measuring tape. Body composition will be assessed in the morning using bioimpedance measurement with a Bodystat Quadscan 4000 Touch (Bodystat, Douglas, Isle of Man, UK) with patients in a supine position after 10 min rest. Electrodes will be connected to the hands (wrist and middle finger) and feet (ankle and above the knuckle of the toe), after those areas are cleaned with alcohol. Using height, mass and sex data, body composition (body fat, lean body mass, total body water) will be calculated from the impedance at different voltages according to the manufacturer's guidelines.

\section{Physical activity and sedentary behaviour}

Physical activity and sedentary behaviour will be assessed using the short form of the International Physical Activity Questionnaire ${ }^{26}$ and an Actigraph wGT3X-BT accelerometer (ActiGraph, Pensacola, Florida, USA) 1 week before inclusion in the CR programme and 1 week after completion. Patients will be instructed on how to wear the accelerometer before attaching it to their right hip using an elastic band. The accelerometer will be worn during usual daily activities for eight consecutive days and be removed only to avoid contact with water (eg, showering). ${ }^{27}$ ActiLife software V.6.13.4 (ActiGraph) will be used for initialisation and extraction of data from the devices. The accelerometer will be initialised for raw mode with a sampling frequency of $100 \mathrm{~Hz}$ at least half an hour after the second day of measurements. The raw acceleration files will be saved to $1 \mathrm{~s}$ epochs and later transferred to a Matlab programme (The MathWorks, Natick, Massachusetts, USA) for further analysis of sedentary behaviour, low physical activity and moderate-to-vigorous physical activity. ${ }^{27}$ The triaxial vector magnitude count per min (VM CPM) (eg, the acceleration units) will be split into 
different physical activity levels as follows: sedentary behaviour (<150 VM CPM), light physical activity (1502689 VM CPM) and moderate-to-vigorous physical activity ( $\geq 2690$ VM CPM). Data with at least 4 days of 10 hours wear time will be included in the final analysis. ${ }^{27}$

\section{Blood biomarkers}

Standard blood samples will be collected at baseline and post-CR and will be immediately analysed for routine purposes (haematological parameters, blood lipids, glucose, glomerulus filtration rate, magnesium, calcium, sodium, potassium, creatinine, N-terminal pro-b-type natriuretic peptide, etc). In addition, samples of plasma and serum will be stored in cryotubes at $-80^{\circ} \mathrm{C}$ within 2 hours of collection for post-hoc analysis of insulin resistance (homoeostatic assessment for insulin resistance, glycated haemoglobin), inflammation (C reactive protein, interleukins, tumour necrosis factor alpha, etc) and growth hormones (growth hormone, testosterone, insulin-like growth factors).

\section{Health-related quality of life and depressive symptoms}

Health-related quality of life will be assessed using the short form 12-item quality of life questionnaire (SF-12) ${ }^{28}$ and psychological well-being (eg, depression) with the patient health 9-item questionnaire (PHQ-9) ${ }^{29}$ at baseline and post-CR. We will calculate the total score of the SF-12, the physical component summary and the mental component summary score. ${ }^{30}$ The SF-12 questionnaire has good internal consistency and good construct validity, ${ }^{31}$ and has been shown to be an excellent alternative to the longer version (36-item quality of life questionnaire) ${ }^{28}$ in patients with CAD. Similarly, the PHQ-9 has shown excellent internal consistency and good construct validity in patients with $\mathrm{CAD}{ }^{32}$

\section{Sample size calculation and statistical analysis}

The GPower V.3.1. programme (University of Dusseldorf, Germany) was used to calculate sample size, based on previously reported mean changes in aerobic capacity or isometric maximal knee-extensor torque when comparing the effects of combined AT with either HL-RT ${ }^{18}$ or LL-RT ${ }^{16}$ with standard care (AT). Assuming a statistical power of $0.80(\beta=0.20)$ and $\alpha=0.05$, a total of 48 patients with $\mathrm{CAD}$ (16 patients per group) need to be enrolled to detect a mean change of $3.30 \mathrm{~mL} / \mathrm{kg} / \mathrm{min}$ (aerobic capacity), ${ }^{18}$ and a total of 12 patients (4 per group) to detect a mean change of $29 \mathrm{Nm}$ (isometric knee strength). ${ }^{16}$ With an expected attrition rate of $10 \%-15 \%,{ }^{33}$ sample size was increased to 60 patients to maintain study power for assessment of primary outcomes. The sample size for comparison between LL-RT and HL-RT was not calculated, as no previous studies were available in patients with CAD. Therefore, in that sense, this study will establish only pilot results on the comparison of HL-RT and LL-RT in combination with AT on coprimary outcomes. Furthermore, statistical power was not calculated for secondary outcomes; thus, all outcomes should be interpreted as hypothesis-generating.

Descriptive statistics will be presented as numbers and percentages for categorical variables and as means and SD or median and IQR (according to the normality of distribution) for numeric variables. Numeric variables will be screened for normality of distribution (ShapiroWilk test), homogeneity of variances (Levene test) and sphericity (Mauchly test). Data will be analysed using the intention-to-treat principle, whereas all patients who completed $>24$ sessions will be included in the final analysis. Between-group differences will be assessed using one-way analysis of variance (ANOVA) or the KruskallWallis test (depending on the assumptions), with additional post-hoc analysis. Training effects will be calculated using two-way ANOVA or analysis of covariance, where appropriate. When data indicate asymmetrical distribution of outcome variables, transformation functions will be applied. Any within-group effect of training intervention will be assessed with paired-samples t-tests or with Wilcoxon tests depending on the normality of distribution. All data will be analysed using IBM SPSS V.25 software (SPSS). The level of statistical significance will be set at alpha $<0.05$.

\section{Ethics and dissemination}

The study design and protocol were approved by the National Medical Ethics Committee of Slovenia (registration number: 0120-573/2019/15), and the study has been registered on ClinicalTrials.gov. The study will be conducted in compliance with the Helsinki Declaration of 1975 (in its most recently amended version) and the American College of Sports Medicine Policy Statement Regarding the Use of Human Subjects and Informed Consent. Any change in the study protocol will be submitted to the National Medical Ethics Committee for consideration and approval. Prior to enrolment, all patients will receive verbal and written information about the study aims, procedures and potential risk during the study and will be asked to sign a written informed consent before beginning study procedures (online supplemental file 1). Written informed consent will be collected by TK. Participation in the study will be voluntary and patients will be able to withdraw from the study at any time and without any consequences. Data on each participant will be anonymised using only ID numbers, and all data entered in electronic databases will be double-checked to ensure quality. Data will be stored in an electronically secured server at the General Hospital Murska Sobota. Only TK and ML will have access to the data and will ensure its integrity. The study will be coordinated by TK and ML. All authors of the study protocol will be involved in study dissemination. The results of the study will be published as peer-reviewed articles and reports, congress presentations, communicated with the clinical community and shared through posts on social media (eg, Twitter and Facebook), independently of the funding agency. Patients will have the possibility of obtaining their 
own results. The results of the study will be disseminated as presentations and practical workshops among national CR centres in Slovenia and within the Slovenian association of coronary clubs. Patients will be invited to share their experience of RT during such events. The combination of patients' experiences together with the results of the study will be used to prepare future feasible exercise training programmes in CR. Data will be shared on reasonable request.

\section{DISCUSSION}

Despite recent progression of exercise prescription and modalities in CR, ${ }^{134} 35$ the implementation of RT into clinical practice remains limited by its heterogeneous prescription, lack of reported progression of training loads and poor reporting of adverse events in the randomised controlled clinical trials. ${ }^{29}$ This study is designed with the aim of establishing whether HL-RT is efficacious and safe compared with currently advised AT with and without the addition of LL-RT.

The study is the first to implement the progressive LL-RT (35\%-40\% of 1-RM) and HL-RT (70\%-80\% of 1-RM) programme with balanced training volume in patients with CAD. Currently, no study supports the dose-dependent relationship between RT load and improvement in aerobic capacity and muscle strength in patients with CAD. In healthy young and older adults such a relationship is well established, as meta-analysis has shown the superior effects of HL-RT on improvement of muscle strength and hypertrophy when compared with LL-RT. ${ }^{11}{ }^{12}$ With the increasing prevalence of frail elderly patients with $\mathrm{CAD}$ enrolled in $\mathrm{CR},{ }^{36}$ we expect that the implementation of HL-RT will have immediate clinical impact especially in such patient groups.

Despite the well-reported safety of RT in patients with cardiovascular disease, ${ }^{29}$ HL-RT was rarely implemented until recent guidelines were published, ${ }^{1}$ probably because of potential cardiovascular complications with excessive increase in blood pressure. ${ }^{737}$ In contrast to this common belief, haemodynamic studies have demonstrated that HL-RE (70\%-90\% of 1-RM) elicits lower heart rate, blood pressure and perceived exertion compared with low-tomoderate RE (35\%-60\% of 1-RM) in patients with CAD with previous training experience in CR. ${ }^{13}{ }^{14}$ To date, no studies have examined haemodynamic responses to RE before its inclusion in CR; thus, our crossover study will be the first to establish the safety of both types of RE. In contrast to previous studies in patients with $\mathrm{CAD},{ }^{13}{ }^{14}$ the exercise load in LL-RE and HL-RE will be balanced to eliminate any potential effects of training intensity, and will measure rating of perceived exertion during the exercise.

The authors acknowledge that the study could be limited by its lack of adequate statistical power to compare the effects of LL-RT and HL-RT on maximal aerobic capacity and muscle strength. In addition, as a consequence of the design of the CR programme, patients cannot be blinded; however, each cluster of randomised patients will train separately to avoid additional comparison with other interventional arms.

In conclusion, we postulate that implementation of HL-RT as an adjunct exercise therapy to AT will help to optimise improvements in aerobic capacity and muscle strength during routine CR programmes.

\section{Author affiliations}

${ }^{1}$ Department of Research and Education, General Hospital Murska Sobota, Murska Sobota, Slovenia

${ }^{2}$ University of Primorska, Faculty of Health Sciences, Izola, Slovenia

${ }^{3}$ Laboratory for Motor Control and Motor Behavior, S2P, Science to Practice,

Ljubljana, Slovenia

${ }^{4}$ Human Health Department, InnoRenew CoE, Izola, Slovenia

${ }^{5}$ Department of Sports Medicine, University of Ljubljana, Faculty of Sport, Ljubljana, Slovenia

${ }^{6}$ Division of Cardiology, General Hospital Murska Sobota, Murska Sobota, Slovenia

${ }^{7}$ University of Ljubljana, Faculty of Medicine, Ljubljana, Slovenia

${ }^{8}$ University of Maribor, Faculty of Natural Sciences and Mathematics, Maribor, Slovenia

\section{Twitter Tim Kambic @TimKambic}

Acknowledgements The authors wish to thank nurses Aleksandra Balažic Gjura, Anita Vogrinčič Černezel, Blanka Rajh and Darija Števančec, and physiotherapists Boža Gider, Maja Lukovnjak, Alen Balažič and Jernej Balažič for their valuable assistance during the recruitment process.

Contributors TK, NS, VH and ML contributed to the conception and/or design and/or planned analysis of the study. TK drafted the manuscript with contributions from $\mathrm{VH}$ and $\mathrm{ML}$. All authors critically revised the manuscript and gave their final approval.

Funding The work is supported by a research fellowship grant (no. 630-72/2019-1) received by TK from the Slovenian Research Agency. ML is funded by the Slovenian Research Agency (grants no. J3-9292, Burden of cachexia and sarcopenia in patients with chronic diseases: epidemiology, pathophysiology and outcomes, and no. J3-9284, Epidemiology, pathophysiology and clinical relevance of anaemia in chronic cardiopulmonary patients). VH is funded by the Slovenian Research Agency (programme grant no. P5-0147). The funding agency has no impact on data collection, analysis or interpretation of the study.

Competing interests The authors report no competing interest within the submitted work. ML reports grants from Roche Diagnostics and personal fees from Vifor Pharma and AstraZeneca outside the submitted work.

Patient and public involvement statement Patients and/or the public were not involved in the design and/or conduct, and/or reporting of this study. Patients will be invited to share their experiences of RT and will be asked to encourage other patients with $C A D$ to enrol into $C R$ programmes within the hospital and/or coronary clubs and associations. The authors will gather patients' experience of RT to structure feasible exercise training programmes with emphasis on RT.

Patient consent for publication Not required.

Provenance and peer review Not commissioned; externally peer reviewed.

Supplemental material This content has been supplied by the author(s). It has not been vetted by BMJ Publishing Group Limited (BMJ) and may not have been peer-reviewed. Any opinions or recommendations discussed are solely those of the author(s) and are not endorsed by BMJ. BMJ disclaims all liability and responsibility arising from any reliance placed on the content. Where the content includes any translated material, BMJ does not warrant the accuracy and reliability of the translations (including but not limited to local regulations, clinical guidelines, terminology, drug names and drug dosages), and is not responsible for any error and/or omissions arising from translation and adaptation or otherwise.

Open access This is an open access article distributed in accordance with the Creative Commons Attribution Non Commercial (CC BY-NC 4.0) license, which permits others to distribute, remix, adapt, build upon this work non-commercially, and license their derivative works on different terms, provided the original work is properly cited, appropriate credit is given, any changes made indicated, and the use is non-commercial. See: http://creativecommons.org/licenses/by-nc/4.0/. 
ORCID iD

Tim Kambic http://orcid.org/0000-0003-3571-7928

\section{REFERENCES}

1 Ambrosetti M, Abreu A, Corr U. Secondary prevention through comprehensive cardiovascular rehabilitation: from knowledge to implementation. 2020 update. A position paper from the secondary prevention and rehabilitation section of the European association of preventive cardiology. Eur J Prev Cardiol 2020.

2 Hollings M, Mavros Y, Freeston J, et al. The effect of progressive resistance training on aerobic fitness and strength in adults with coronary heart disease: a systematic review and meta-analysis of randomised controlled trials. Eur J Prev Cardiol 2017;24:1242-59.

3 Anderson L, Oldridge N, Thompson DR, et al. Exercise-Based Cardiac Rehabilitation for Coronary Heart Disease. J Am Coll Cardiol 2016;67:1-12.

4 Keteyian SJ, Brawner CA, Savage PD, et al. Peak aerobic capacity predicts prognosis in patients with coronary heart disease. Am Heart J 2008;156:292-300.

5 Price KJ, Gordon BA, Bird SR, et al. A review of guidelines for cardiac rehabilitation exercise programmes: is there an international consensus? Eur J Prev Cardiol 2016;23:1715-33.

6 Verrill DE, RibisI PM. Resistive exercise training in cardiac rehabilitation. An update. Sports Med 1996;21:347-83.

7 Bjarnason-Wehrens B, Mayer-Berger W, Meister ER, et al. Recommendations for resistance exercise in cardiac rehabilitation. recommendations of the German Federation for cardiovascular prevention and rehabilitation. Eur J Cardiovasc Prev Rehabil 2004;11:352-61.

8 Williams MA, Haskell WL, Ades PA, et al. Resistance exercise in individuals with and without cardiovascular disease: 2007 update: a scientific statement from the American heart association Council on clinical cardiology and Council on nutrition, physical activity, and metabolism. Circulation 2007;116:572-84.

9 Xanthos PD, Gordon BA, Kingsley MIC. Implementing resistance training in the rehabilitation of coronary heart disease: a systematic review and meta-analysis. Int J Cardiol 2017;230:493-508.

10 American College of Sports Medicine. American College of sports medicine position stand. progression models in resistance training for healthy adults. Med Sci Sports Exerc 2009;41:687-708.

11 Raymond MJ, Bramley-Tzerefos RE, Jeffs KJ, et al. Systematic review of high-intensity progressive resistance strength training of the lower limb compared with other intensities of strength training in older adults. Arch Phys Med Rehabil 2013;94:1458-72.

12 Schoenfeld BJ, Grgic J, Ogborn D, et al. Strength and hypertrophy adaptations between low- vs. High-Load resistance training: a systematic review and meta-analysis. J Strength Cond Res 2017;31:3508-23.

13 Gjovaag TF, Mirtaheri P, Simon K, et al. Hemodynamic responses to resistance exercise in patients with coronary artery disease. Med Sci Sport Exerc 2016;48:581-8.

14 Lamotte M, Niset G, van de Borne P. The effect of different intensity modalities of resistance training on beat-to-beat blood pressure in cardiac patients. Eur J Cardiovasc Prev Rehabil 2005;12:12-17.

15 Schulz KF, Altman DG, Moher D, et al. Consort 2010 statement: updated guidelines for reporting parallel group randomised trials. BMJ 2010;340:c332.

16 Gayda M, Choquet D, Ahmaidi S. Effects of exercise training modality on skeletal muscle fatigue in men with coronary heart disease. J Electromyogr Kinesiol 2009;19:e32-9 http://www. sciencedirect.com/science/article/pii/S1050641107001502

17 Baechle TR, Earle RW, Wathen D. Resistance Training. In: Baechle TR, Earle RW, eds. Essentials of strength and conditioning Researchq. Champagne, Illinois: Human Kinetics, 2008: 381-412.
18 Marzolini S, Oh PI, Thomas SG, et al. Aerobic and resistance training in coronary disease: single versus multiple sets. Med Sci Sports Exerc 2008;40:1557-64.

19 Lamotte M, Fleury F, Pirard M, et al. Acute cardiovascular response to resistance training during cardiac rehabilitation: effect of repetition speed and rest periods. Eur J Cardiovasc Prev Rehabil 2010;17:329-36.

20 Fletcher GF, Ades PA, Kligfield P, et al. Exercise standards for testing and training: a scientific statement from the American heart association. Circulation 2013;128:873-934.

21 Bily W, Sarabon N, Löfler S, et al. Relationship between strength parameters and functional performance tests in patients with severe knee osteoarthritis. Pm R 2019;11:834-42.

22 Brzycki M. Strength Testing-Predicting a One-Rep max from Repsto-Fatigue. J Phys Educ Recreat Dance 1993;64:88-90.

23 Borg GAV. Psychophysical bases of perceived exertion. Medicine \& Science in Sports \& Exercise 1982;14:377???381-81 http://www. ncbi.nlm.nih.gov/pubmed/7154893

24 Guralnik JM, Seeman TE, Tinetti ME, et al. Validation and use of performance measures of functioning in a non-disabled older population: MacArthur studies of successful aging. Aging Clin Exp Res 1994;6:410-9.

25 Rikli RE, Jones CJ. Development and validation of a functional fitness test for community-residing older adults. J Aging Phys Act 1999;7:129-61.

26 Craig CL, Marshall AL, Sjöström M. International physical activity questionnaire: 12-country reliability and validity. Medicine \& Science in Sports \& Exercise 2003;35:1381-95.

27 Sagelv EH, Ekelund U, Pedersen S, et al. Physical activity levels in adults and elderly from triaxial and uniaxial accelerometry. The Tromsø study. PLoS One 2019;14:e0225670.

28 Müller-Nordhorn J, Roll S, Willich SN. Comparison of the short form (SF)-12 health status instrument with the SF-36 in patients with coronary heart disease. Heart 2004;90:523-7.

29 Kroenke K, Spitzer RL, Williams JB. The PHQ-9: validity of a brie depression severity measure. J Gen Intern Med 2001;16:606-13.

30 Ware J, Kosinski M, Keller SD. A 12-Item short-form health survey: construction of scales and preliminary tests of reliability and validity. Med Care 1996;34:220-33.

31 De Smedt D, Clays E, Doyle F, et al. Validity and reliability of three commonly used quality of life measures in a large European population of coronary heart disease patients. Int J Cardiol 2013;167:2294-9 https://www.sciencedirect.com/science/article/pii/ S0167527312008005

32 Stafford L, Berk M, Jackson HJ. Validity of the hospital anxiety and depression scale and patient health Questionnaire- 9 to screen for depression in patients with coronary artery disease. Gen Hosp Psychiatry 2007;29:417-24 https://www.sciencedirect.com/science/ article/pii/S0163834307001296

33 Pardaens S, Willems A-M, Clays E, et al. The impact of drop-out in cardiac rehabilitation on outcome among coronary artery disease patients. Eur J Prev Cardiol 2017;24:1490-7.

34 Vanhees L, Geladas N, Hansen D, et al. Importance of characteristics and modalities of physical activity and exercise in the management of cardiovascular health in individuals with cardiovascular risk factors: recommendations from the EACPR (Part II). Eur J Prev Cardiol 2012;19:1005-33.

35 Pattyn N, Beulque R, Cornelissen V. Aerobic interval vs. continuous training in patients with coronary artery disease or heart failure: an updated systematic review and meta-analysis with a focus on secondary outcomes. Sports Medicine 2018;48:1189-205.

36 Vigorito C, Abreu A, Ambrosetti M, et al. Frailty and cardiac rehabilitation: a call to action from the EAPC cardiac rehabilitation section. Eur J Prev Cardiol 2017;24:577-90.

37 Angius L, Crisafulli A. Exercise intolerance and fatigue in chronic heart failure: is there a role for group III/IV afferent feedback? Eur J Prev Cardiol 2020;27:1862-72. 